\title{
Evaluation of Transforming Growth Factor Beta-1 Gene 869T/C Polymorphism with Hypertension: A Meta-Analysis
}

\author{
Wenquan $\mathrm{Niu}^{1,2,3}$ \\ ${ }^{1}$ State Key Laboratory of Medical Genomics, Shanghai Key Laboratory of Vascular Biology, and Department of Hypertension, \\ Ruijin Hospital, Shanghai Jiao Tong University School of Medicine, Ruijin Second Road 197, Shanghai 200025, China \\ ${ }^{2}$ Laboratory of Vascular Biology, Institute of Health Sciences, Shanghai Institutes for Biological Sciences, Chinese Academy of Sciences, \\ Chongqing South Road 225, Shanghai 200025, China \\ ${ }^{3}$ Shanghai Institute of Hypertension, Shanghai Jiao Tong University School of Medicine, Ruijin Second Road 197, \\ Shanghai 200025, China
}

Correspondence should be addressed to Wenquan Niu, niuwenquan@yahoo.cn

Received 14 March 2011; Revised 8 May 2011; Accepted 11 May 2011

Academic Editor: Cesare Cuspidi

Copyright (C) 2011 Wenquan Niu. This is an open access article distributed under the Creative Commons Attribution License, which permits unrestricted use, distribution, and reproduction in any medium, provided the original work is properly cited.

\begin{abstract}
Association between transforming growth factor beta-1 gene (TGFB1) 869T/C polymorphism and hypertension has been widely evaluated, yet with conflicting results. As meta-analysis is a reliable way to resolve discrepancies; I aimed to evaluate this association. Data were available from 9 study populations involving 6151 subjects. Overall, comparison of allele 869C with 869T generated a significant $30 \%$ increased hypertension risk (95\% confidence interval [95\% CI]: 1.11-1.51; $P=0.001$ ), which was strengthened for homozygous comparison (869CC versus 869TT) with odds ratio (OR) doubled to 1.62 (95\% CI: $1.23-2.14 ; P=0.001$ ). Stratified analysis by study design demonstrated stronger associations in population-based studies than in hospital-based studies with OR, except in the dominant model, being increased by 7.94-18.61\%. Likewise, ethnicity-based analysis exhibited a contradictory association between Asians and Whites. Conclusively, these findings support the notion that TGFB1 gene $869 \mathrm{~T} / \mathrm{C}$ polymorphism may influence the risk of hypertension, especially in Asian populations.
\end{abstract}

\section{Introduction}

Hypertension is a complex multifactorial disorder with masses of genetic and environmental factors contributing to its occurrence [1]. Although great effort has been devoted to uncover the genetic underpinnings of hypertension, there is no definite consensus on how many genes and which genetic determinants are actually involved in its development.

Currently, evidence that links inflammation to the genesis of hypertension is proliferating $[2,3]$. As an antiinflammatory regulator, transforming growth factor beta-1 or TGF- $\beta 1$ (gene: TGFB1) plays a part in many different clinical processes, such as embryonal development, cellular proliferation and differentiation, wounding healing, and angiogenesis $[4,5]$. In addition, augmented production of TGF- $\beta 1$, partly via the mediation of angiotensin II, potentially contributes to target organ damage related to hypertension [6-9].
Since the genomic sequence of TGFB1 gene is highly polymorphic, it is of added interest to confirm which TGFB1 polymorphism(s) might have functional potentials to influence the final bioavailability of TGF- $\beta 1$, thus the development of hypertension. In particular, an exonic polymorphism, 869T/C (rs1982073) in TGFB1 gene, has been studied extensively; however, the results are not often reproducible with positive signals being reported in some [10], but not all $[11,12]$, studies. Generally, association studies with individually low statistical power might account for this lack of consistency [13].

As meta-analysis is a reliable way to resolve discrepancies in association studies and in an effort to clarify earlier inconclusive results, I decided to evaluate the influence of TGFB1 gene 869 T/C polymorphism on the occurrence of hypertension, while addressing between-study heterogeneity, as well as publication bias. 


\section{Methods}

2.1. Literature Search. Both English and Chinese language publications were identified using PubMed and EMBASE engines, as well as China Biological Medicine (http://sinomed.imicams.ac.cn/index.jsp) and Wanfang (http://www.wanfangdata.com.cn) databases with the deadline at February 1, 2011. Keywords used for search in the Boolean expression were (transforming growth factor beta-1 OR TGF$\beta 1$ OR TGFB1) AND (hypertension OR blood pressure) AND (polymorphism OR allele OR genotype OR variant OR variation). Searching results were limited to human populations (rather than family-based populations). The full text of the retrieved articles was scrutinized to decide whether information on the topic of interest was included. In addition, reference lists of the retrieved articles and reviews were also checked for citations of publications that were not initially identified. If more than one geographic or ethnic groups were included in one publication, each group was treated separately.

2.2. Inclusion/Exclusion Criteria. Qualified studies in this meta-analysis met the following criteria: (i) evaluation of the TGFB1 gene 869T/C polymorphism with hypertension; (ii) case-control or cross-sectional study using either a hospitalbased or population-based design; (iii) sufficient information on $869 \mathrm{~T} / \mathrm{C}$ genotype counts between hypertensive patients and controls for estimating odds ratio (OR) and its corresponding 95\% confidence interval (95\% CI).

Hypertension was defined as systolic blood pressure equal to or above $140 \mathrm{mmHg}$ or diastolic blood pressure equal to or above $90 \mathrm{mmHg}$ or previous treatment with antihypertensive drugs. Studies evaluating secondary hypertension or other types of monogenic hypertension were excluded. Where there were multiple publications from the same study population, the most complete and recent results were extracted.

2.3. Extracted Information. The following information was extracted from each qualified study: first author's name, publication date, population ethnicity, study design, diagnostic criteria, baseline characteristics of the study population (such as age, gender, and body mass index), and the 869T/C genotype counts in patients and controls. For consistency, continuous variables expressed as mean \pm standard error (SE) were converted to mean \pm standard deviation (SD). Moreover, the units of measures used in this study are transformed into the standard measurement units. Data and study quality were assessed in duplicate.

2.4. Statistical Analysis. In this meta-analysis, I assessed the association of TGFB1 gene $869 \mathrm{C}$ allele with hypertension relative to the $829 \mathrm{~T}$ allele (allelic model), as well as the homozygous contrast (869CC versus 869TT), the dominant model (869CC plus $869 \mathrm{TC}$ versus $869 \mathrm{TT}$ ), and the recessive model (869CC versus 869TT plus 869TC). Unadjusted OR and $95 \%$ CI were used to compare contrasts of alleles or genotypes between patients and controls. The randomeffects model using the method of DerSimonian \& Laird, instead of fixed-effects model, was implemented to bring the individual effect-size estimates together, and the estimate of heterogeneity was taken from the Mantel-Haenszel model [14].

Satisfaction of 869T/C genotypes with Hardy-Weinberg proportions was calculated using the $\chi^{2}$ test or Fisher's exact test in control groups. Between-study heterogeneity was assessed by the inconsistency index $I^{2}$ statistic (ranging from 0 to $100 \%$ ), which was documented for the percentage of the observed between-study variability due to heterogeneity rather than chance, with higher values of this index suggesting the existence of heterogeneity $[15,16]$. In the case of between-study heterogeneity, I examined the study characteristics that can stratify the studies into subgroups with homogeneous effects.

Cumulative meta-analysis was conducted to identify the influence of the first published study on the subsequent publications and the evolution of the combined estimates over time according to the ascending date of publication [17]. Likewise to identify potentially influential studies, sensitivity analysis was undertaken by removing an individual study each time to check whether any of these estimates can bias the overall estimate.

Additionally, to estimate the extent to which one or more covariates explain heterogeneity, metaregression, as an extension to random-effects meta-analysis, was employed. The metaregression model relates the treatment effect to the study-level covariates including averaged values of age, male percent, body mass index (BMI), glucose, triglyceride (TG), total cholesterol (TC), high-density lipoprotein cholesterol (HDLC), and low-density lipoprotein cholesterol (LDLC) between patients and controls, as well as the study design (population-based design versus hospital-based design) and ethnicity (Asians versus Whites).

I used the funnel plots and Egger regression asymmetry test to examine publication bias. Egger's test can detect funnel plot asymmetry by determining whether the intercept deviates significantly from zero in a regression of the standardized effect estimates against their precision [18].

Probability less than 0.05 was judged as significant with the exception of the $I^{2}$ statistic and publication test, where a significance level of less than 0.1 was chosen. Data management and statistical analyses were performed using STATA version 11.0 for Windows.

\section{Results}

3.1. Description of Available Studies. The initial literature search yielded 66 publications. Further application of the identification criteria left 8 published papers [10-12, 1923] involving 9 study populations (case-patients/controls: $2747 / 3404)$ in an attempt to evaluate the association of TGFB1 gene 869T/C polymorphism with hypertension. Thereof, three papers were written in English language [1012], and the remaining in Chinese language. The study aims and main results of Chinese language reports are presented 


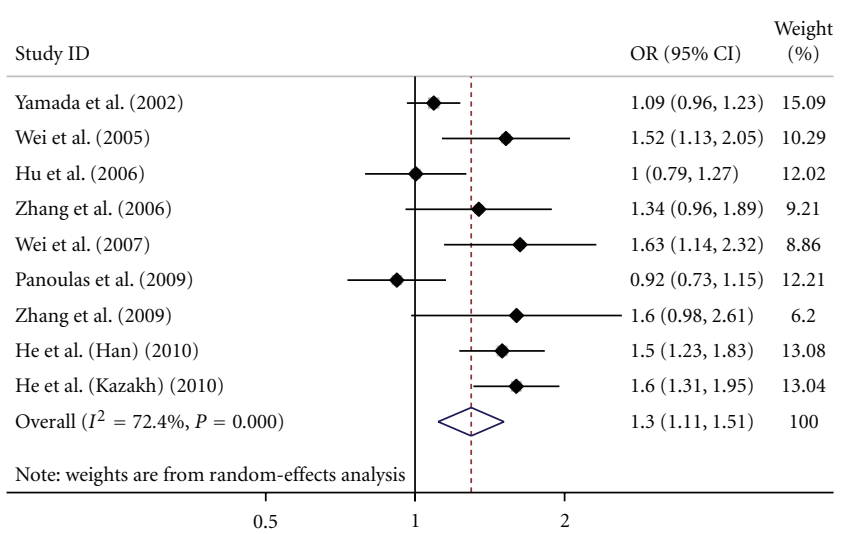

(a)

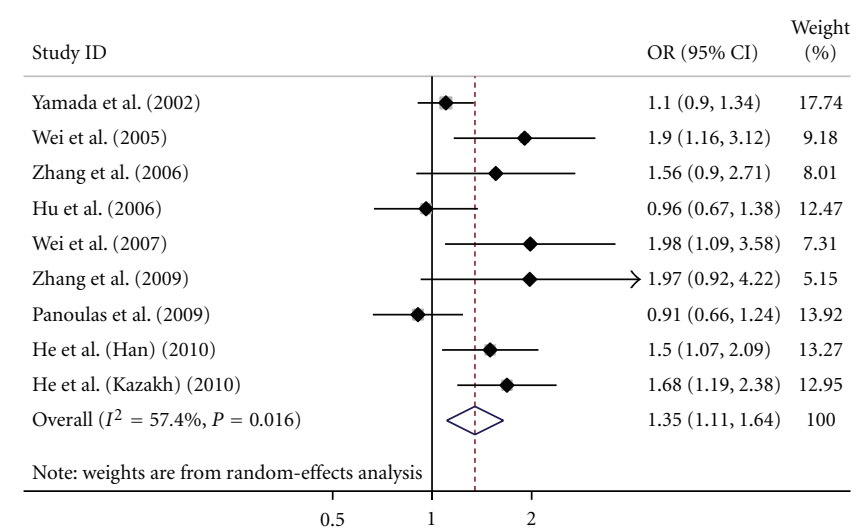

(c)

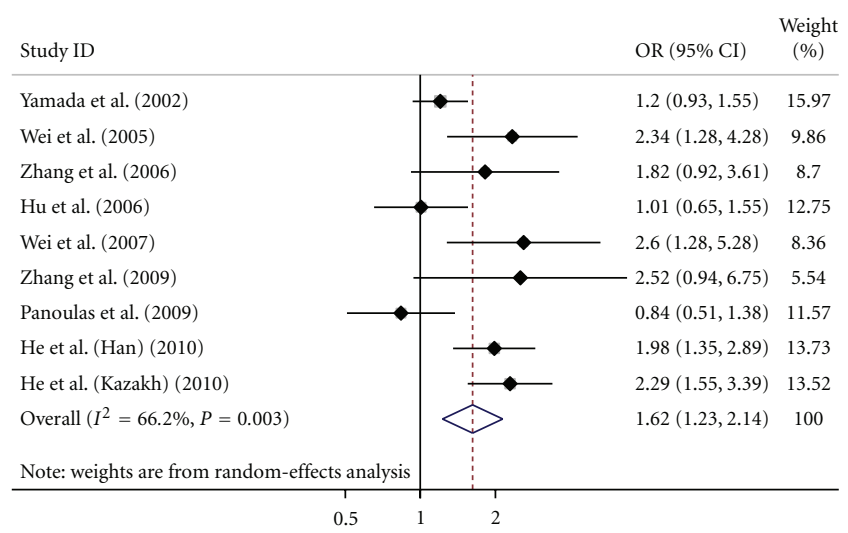

(b)

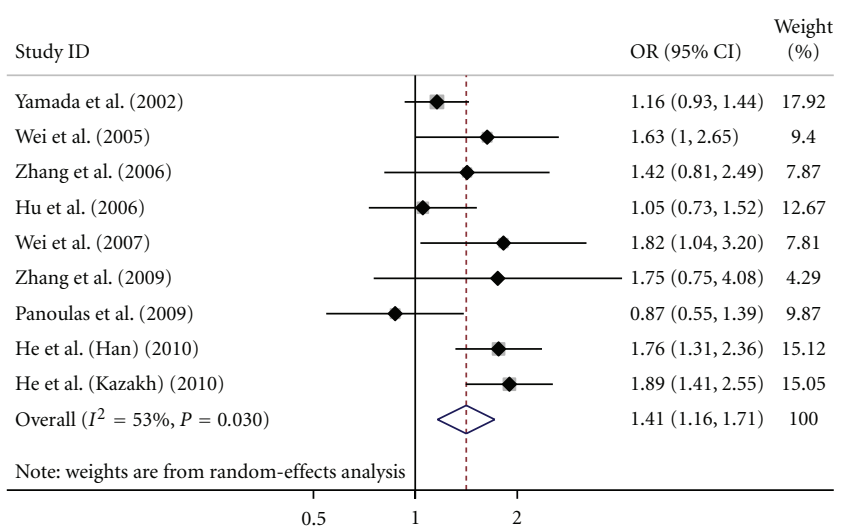

(d)

FIGURE 1: Overall risk estimates of TGFB1 gene 869T/C polymorphism for hypertension in the allelic (a), homozygous (b), dominant (c), and recessive (d) models.

in Supplementary Table 1 (see Table 1 in Supplementary Material available online at doi:10.4061/2011/934265). Seven populations included Chinese subjects [10, 19-23], one included Japanese subjects [11], and one included White population [12]. Except one study [12] involving patients with both hypertension and rheumatoid arthritis, others were focusing on essential hypertension patients. The baseline characteristics of the study populations are summarized in Table 1.

Genotyping for 869T/C polymorphism in all qualified studies, except one [12] using Roche LightCycler method, was conducted using polymerase chain reaction-restriction fragment length polymorphism (PCR-RFLP) followed by enzyme digestion. The frequencies of $869 \mathrm{C}$ allele in the case/control groups were $0.527 / 0.457$ in all populations and were exceedingly low $(0.347 / 0.367)$ in Whites. Taking into account only the control groups, genotype distributions were in Hardy-Weinberg equilibrium across all studies.

3.2. Overall Analysis. In allelic model, comparison of the mutant $869 \mathrm{C}$ allele with the wild $869 \mathrm{~T}$ allele generated a significant 30\% increased risk for hypertension (95\% CI: $1.11-1.51 ; P=0.001)$, yet with strong evidence of between-study heterogeneity $\left(I^{2}=72.4 \% ; P<0.0005\right)$ (Figure 1). Besides the suggestive symmetry of funnel plot
(Figure 2), Egger's test indicated no publication bias $(P=$ $0.25)$. Further, this association was potentially strengthened in the homozygous comparison (869CC versus 869TT) with OR nearly doubled to 1.62 (95\% CI: $1.23-2.14 ; P=0.001$ ). Similarly, this association was still tingled by significant heterogeneity $\left(I^{2}=66.2 \% ; P=0.003\right)$, the risk estimates from individual studies were symmetric (Figure 2), and the Egger's test suggested a low probability of publication bias $(P=0.262)$.

Additionally, in view of the heterozygous 869TC genotype, I considered two different models of inheritance. Overall, the ORs from allelic model were almost similar in magnitude from both dominant $(\mathrm{OR}=1.35 ; 95 \% \mathrm{CI}$ : $1.11-1.64 ; P=0.003)$ and recessive $(\mathrm{OR}=1.41 ; 95 \%$ CI: $1.16-1.71 ; P<0.0005)$ models (Figure 1). Although between-study heterogeneity was attenuated to a certain extent, statistical tests still reached significance (dominant: $I^{2}=57.4 \% ; P=0.016$; recessive: $I^{2}=53.0 \% ; P=$ $0.03)$. Moreover, there was evident publication bias only in dominant model as reflected by the funnel plot and statistical test $(P=0.06)$.

3.3. Cumulative and Sensitivity Analyses. In the cumulative meta-analysis, there was no evidence suggesting the first published study that reported a potentially significant result 


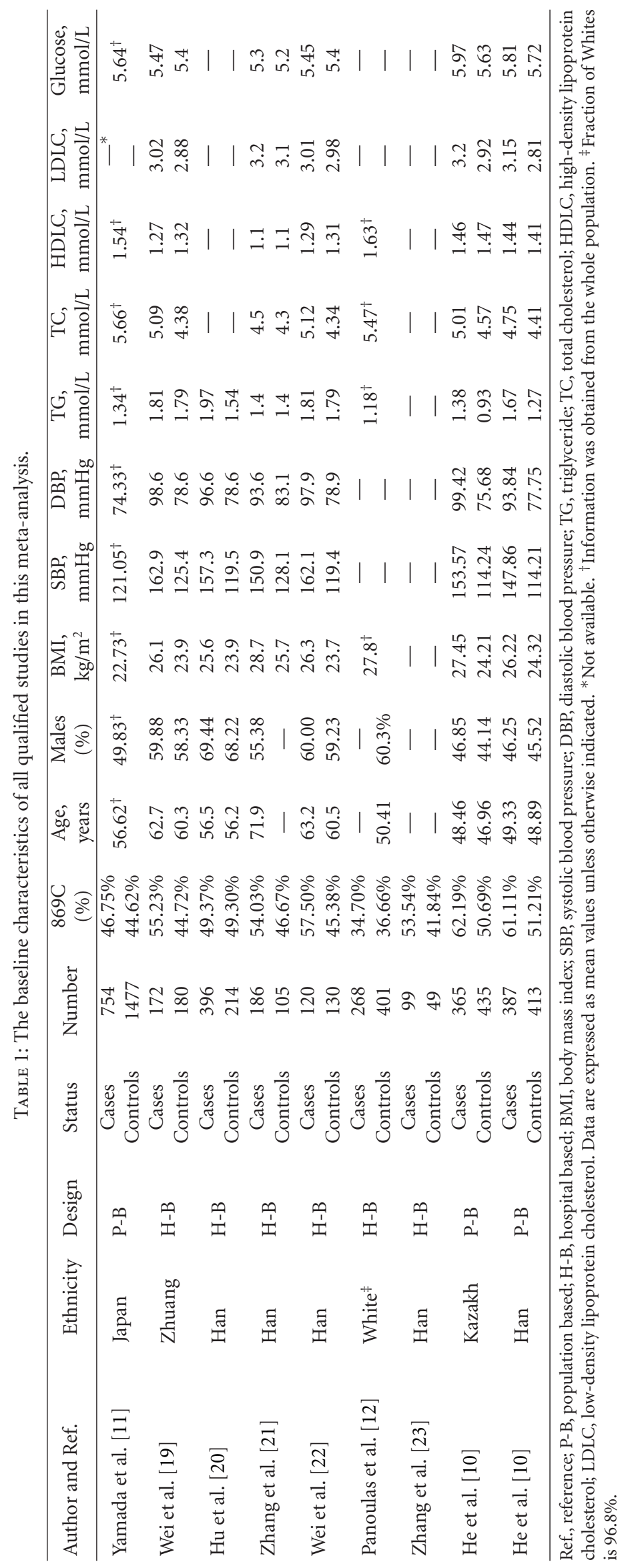




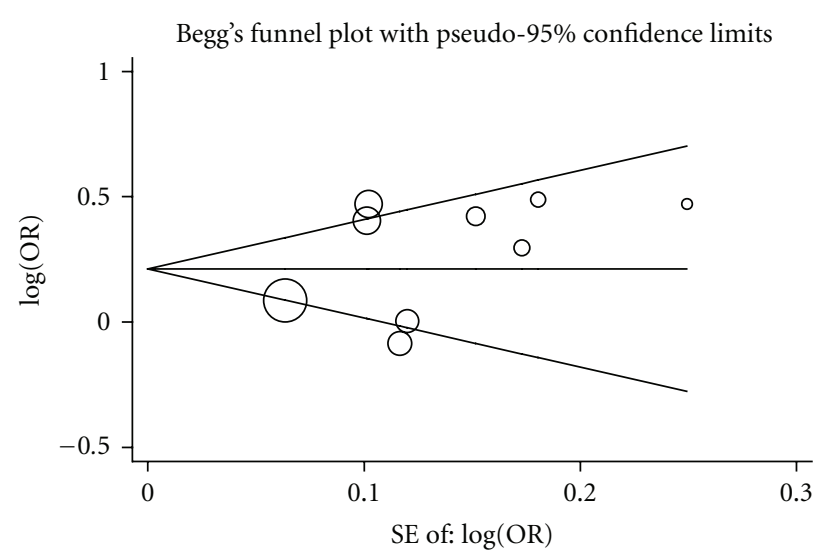

(a)

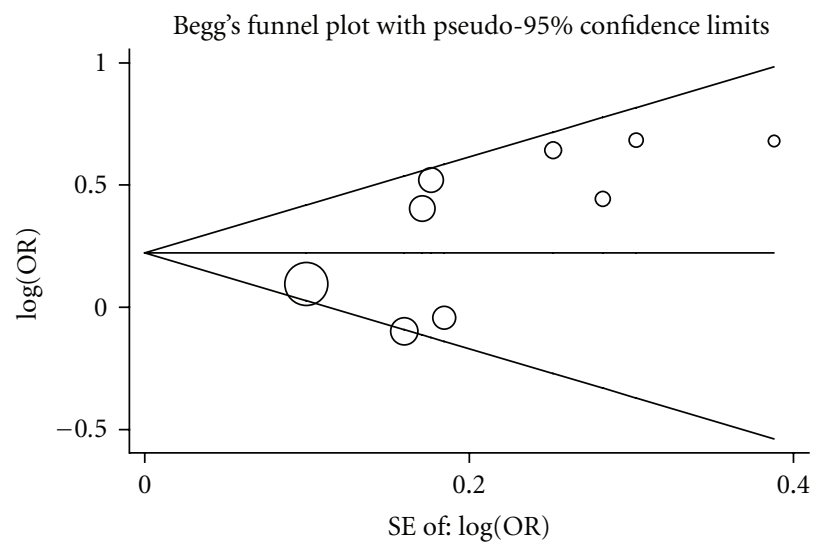

(c)

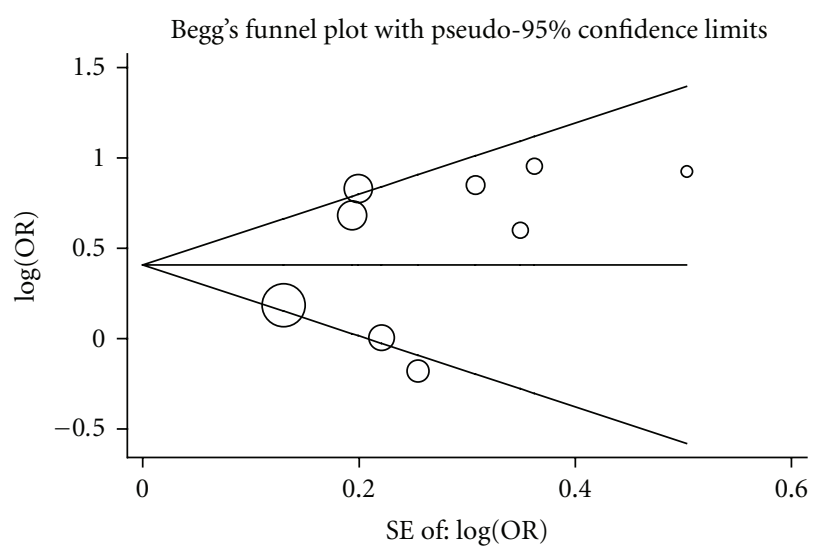

(b)

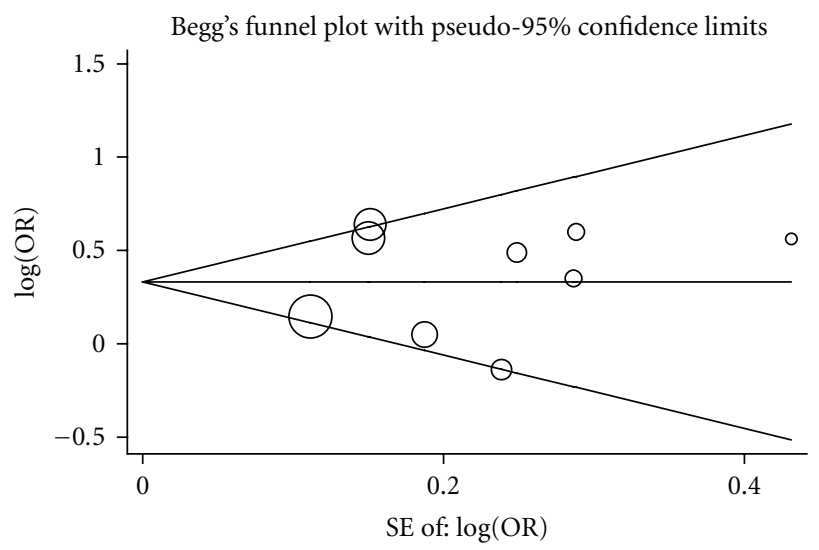

(d)

FIGURE 2: Funnel plots for studies investigating the effect of TGFB1 gene 869T/C polymorphism on the risk of hypertension across the allelic (a), homozygous (b), dominant (c), and recessive (d) models. Vertical axis represents the log of OR; horizontal axis represents the SE of $\log (\mathrm{OR})$. Funnel plots are drawn with $95 \%$ confidence limits. OR, odds ratio; SE, standard error. The graphic symbols represent the data in the plot sized proportional to the inverse variance.

and then trigged the subsequent replication. Also generally the sensitivity analysis revealed that no single studies were observed to influence the pooled results significantly (data not shown).

3.4. Sources of Heterogeneity. Considering the significant heterogeneity in the above comparisons, I considered it a better choice to try investigating its sources by first conducting subgroup analyses in homogeneous groups and then incorporating various study-level covariates in a metaregression model. To evaluate the possible effect of study design on the variability of overall estimates, studies were divided into population based and hospital based, and importantly the magnitude of association in population-based studies was gradiently potentiated in $869 \mathrm{~T} / \mathrm{C}$ allelic (OR increased by $7.94 \%)$, homogeneous $(10.13 \%)$, and recessive (18.61\%) models compared with that in hospital-based studies (Figure 3).

Further ethnicity-stratified analysis indicated strikingly heterogeneous associations of $869 \mathrm{~T} / \mathrm{C}$ polymorphism with hypertension, by showing a contradictory association between Asians and Whites (Figure 4). In Asians, I consistently observed a risk-conferring effect of the 869C allele or 869CC genotype for hypertension, even upon stratification by countries, as well as Chinese ethnic groups. Contrastingly, after restricting analysis to Whites, although there was just one study [12], I hereto observed a protective effect $(\mathrm{OR}=0.84-0.92)$, and the corresponding wide confidence intervals in all genetic models gave an indication of insufficient study power in White populations.

After metaregressing the explanatory variables of interest in this study (see Section 2.4), I unfortunately failed to detect any statistical significance concerning $869 \mathrm{~T} / \mathrm{C}$ polymorphism across all genetic models (data not shown).

\section{Discussion}

Via a comprehensive evaluation of TGFB1 gene 869T/C polymorphism among 6151 subjects, I provided for the first time convincing evidence that individuals homozygous for the $869 \mathrm{C}$ allele were $62 \%$ more likely to develop hypertension with respect to homozygous for the 869TT subjects. Although between-study heterogeneity, albeit disturbing, could not be easily eliminated, this study indicated that TGFB1 gene could be a genetic marker for hypertension. 


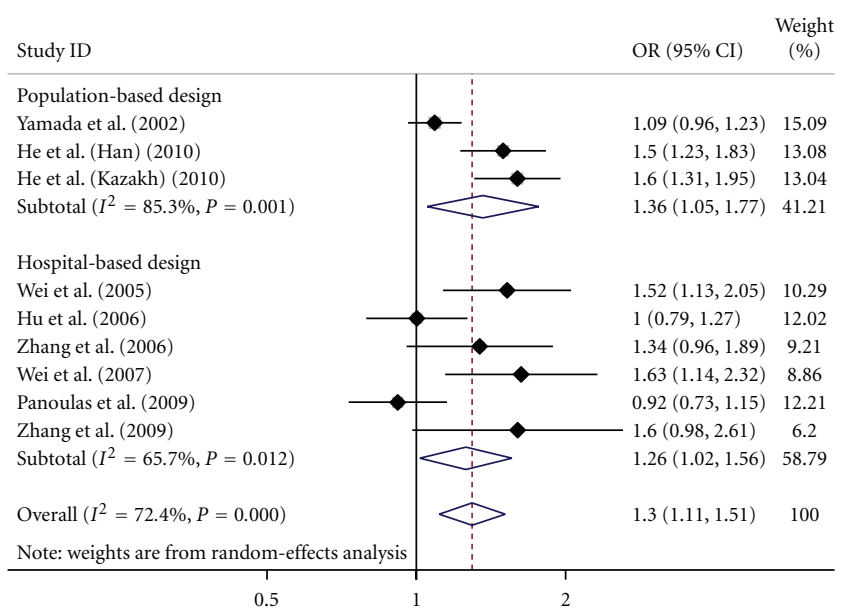

(a)

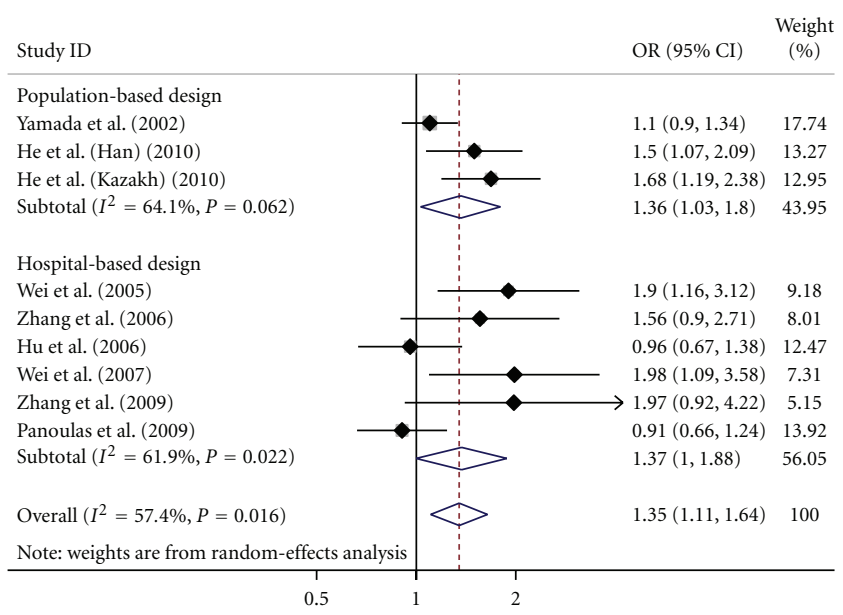

(c)

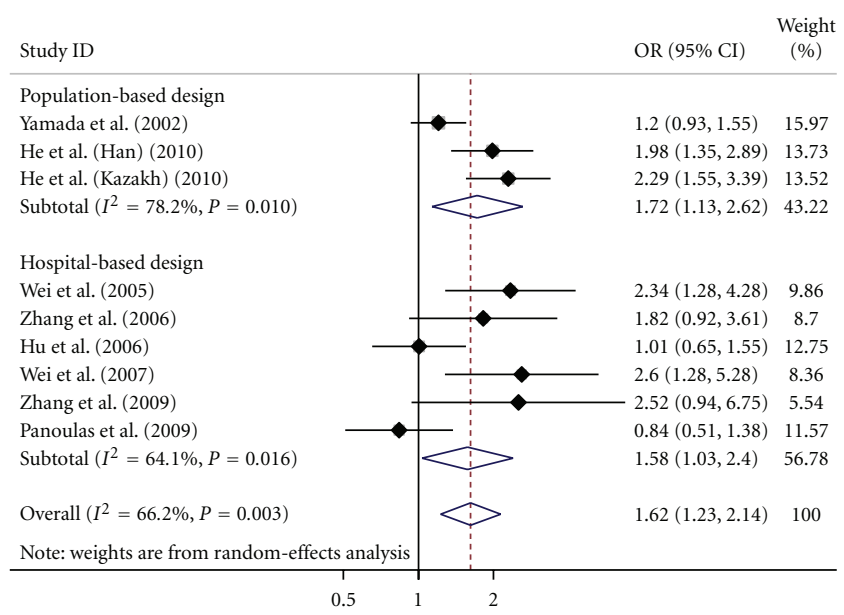

(b)

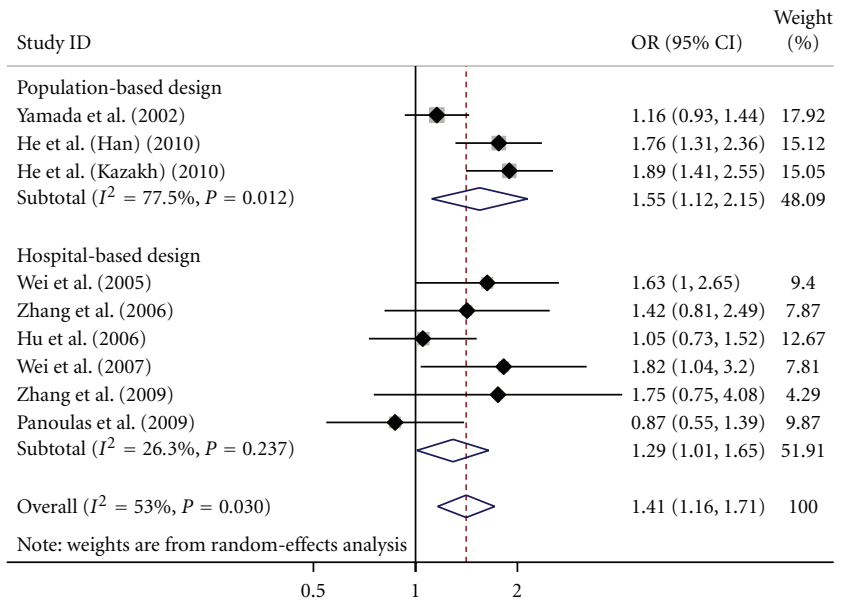

(d)

FIGURE 3: Stratified analysis of TGFB1 gene 869T/C polymorphism by study design with hypertension in the allelic (a), homozygous (b), dominant (c), and recessive (d) models.

In this meta-analysis, study design and ethnicity were regarded as potential sources of between-heterogeneity by subgroup but not metaregression analyses. Although I only focused on nine populations, which runs the risk of falsepositive findings, my results can still drop several hints here. Firstly, subgroup analysis indicated that magnitude of association was potentially strengthened in population-based studies relative to in hospital-based studies. I agree that control for population stratification remains an important consideration in hospital-based studies [24], because in this meta-analysis, most studies have recruited subjects from only one hospital, and thus there might be a narrow socioeconomic profile for both patients and controls. Moreover, in hospital-based studies, poor comparability between cases and controls might exert a confounding effect on the true association in light of a regional specialty for the disease under study and the differential hospitalization rates between cases and controls [25]. In contrast, subjects drawn from community or a fixed group might be representative of the true population, leading us to believe that results from population-based studies might hold the water. Considering the wider confidence intervals of estimates and small sample sizes in population-based studies, more studies are required to quantify this effect size reliably.

Secondly, remarkable heterogeneous associations of 869T/C polymorphism with hypertension were identified across different ethnic populations. Several factors might contribute to this phenomenon. On one hand, hypertension is a complex disease, and different genetic profiles may cause this discrepancy, as indicated by the big difference of $869 \mathrm{C}$ allele frequencies across different populations. In this regard, it is important to construct a database of genetic variants related to hypertension in each ethnic group [26]. On the other hand, this discrepancy is likely due to chance because there is only one study in Whites, which might be statistically underpowered to detect a slight effect or may have generated a fluctuated risk estimate. It is thus obvious that more studies are required in subjects of Caucasian descent in order to fully address this issue.

Thirdly, as an alternative approach to subgroup analysis and a multivariate meta-analysis, the metaregression failed to provide any significant signals regarding the allelic/genotypic 


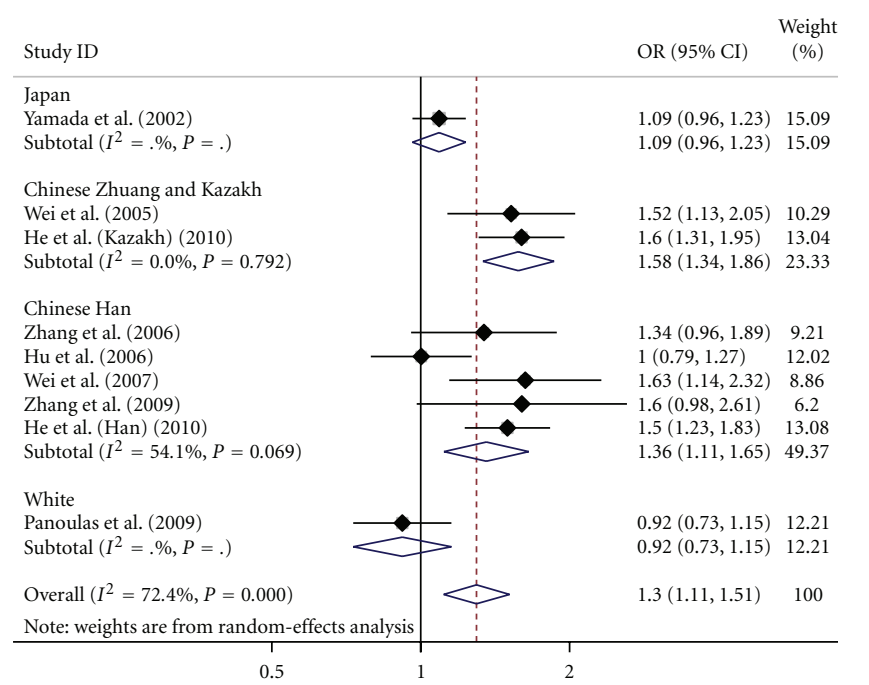

(a)

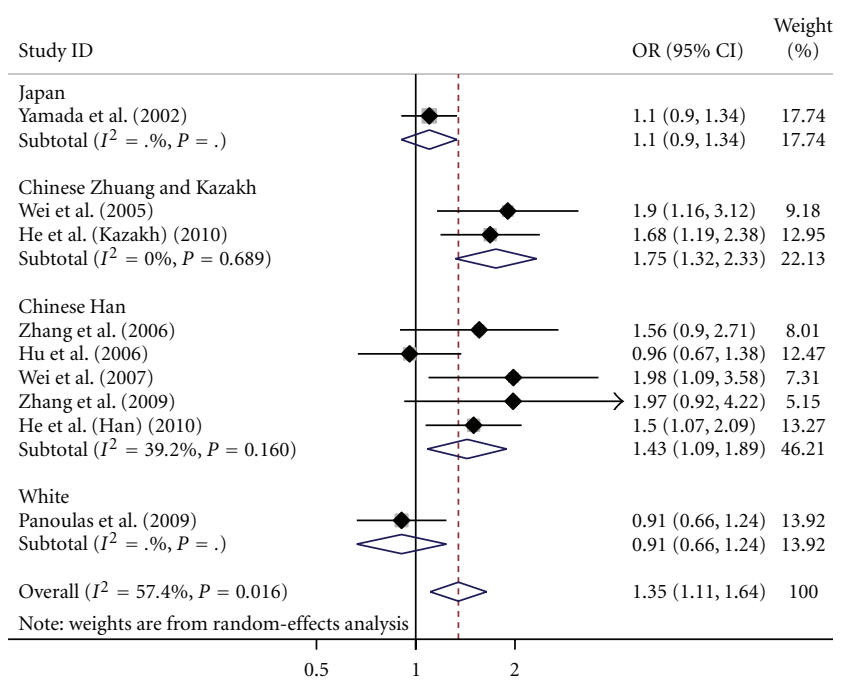

(c)

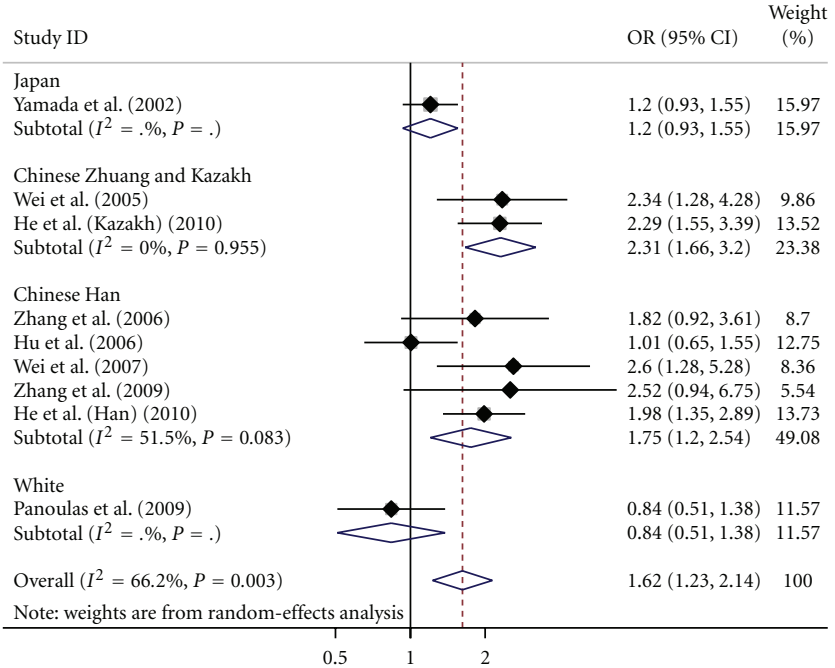

(b)

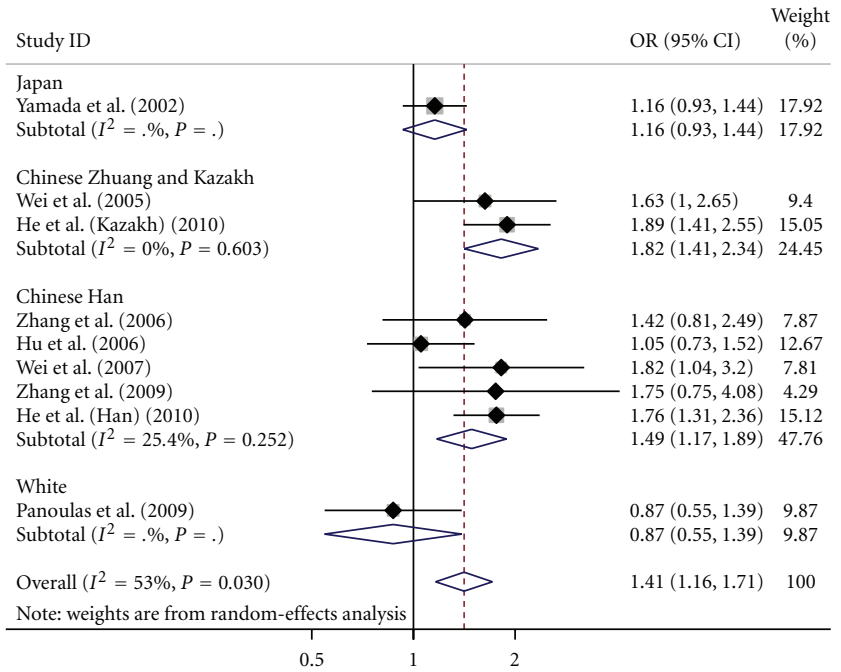

(d)

FIGURE 4: Stratified analysis of TGFB1 gene 869T/C polymorphism by ethnicity with hypertension in the allelic (a), homozygous (b), dominant (c), and recessive (d) models.

associations of $869 \mathrm{~T} / \mathrm{C}$ polymorphism with hypertension. However, it is important to bear in mind that metaregression analysis, although enabling covariates to be considered, does not have the methodological rigor of a properly designed study that is intended to test the effect of these covariates formally [27]. Importantly, one limitation tarnishing this meta-analysis was the number of studies that are available for inclusion. In fact, some studies did not report the study-level covariates of interest, precluding a more robust assessment of sources of heterogeneity.

Last but not least, despite the clear strength of this study including relatively large sample sizes, satisfaction of Hardy-Weinberg equilibrium, and lack of publication bias, interpretation of the current study, however, should be viewed in light of several technical limitations. Because only published studies were retrieved in this meta-analysis and the "grey" literature (papers in languages other than English and Chinese) was not included, publication bias might be possible, even though the funnel plots and statistical tests did not show it. In addition, most studies in this metaanalysis have recruited subjects aged $\geq 50$ years, for whom environmental factors are likely to contribute more prominently than the genetic component to the development of hypertension [28], suggesting that large association studies in a younger population of hypertensive subjects are of added interest. Moreover, the single-locus-based nature of metaanalysis precluded the possibility of gene-gene and geneenvironment interactions, as well as haplotype-based effects, suggesting that additional studies assessing these aspects will be necessary. Furthermore, I only centered on TGFB1 gene $869 \mathrm{~T} / \mathrm{C}$ polymorphism and did not covered other genes or polymorphisms. It seems likely that the $869 \mathrm{~T} / \mathrm{C}$ polymorphism individually makes a moderate contribution to risk prediction in hypertensive subjects, but whether this variant integrated with other risk factors will enhance the prediction requires additional research. Thus, the jury 
must refrain from drawing a conclusion until large, wellperformed studies confirm or refuse this result.

Taken together, I expand previous individual findings on hypertension, indicating that the TGFB1 gene $869 \mathrm{~T} / \mathrm{C}$ polymorphism may influence the risk of hypertension, especially in Asian populations. Also my observation leaves open the question of heterogeneous effect of $869 \mathrm{C}$ allele across different ethnic groups. I believed that this study provides an anchoring point for better understanding of the pathogenesis of hypertension. Nevertheless, for practical reasons, I hope that this study will not remain just another endpoint of research instead of a beginning to establish the background data for further investigation on mechanisms of the TGFB1 gene and hypertension.

\section{Acknowledgments}

This work was supported by the SMC-Excellent Young Faculty Award of Shanghai Jiao Tong University, the Youth Foundation of Shanghai Municipal Health Bureau (2010Y050), the Shanghai “Chen Guang” Project (09CG12), the Shanghai Rising Star Program (11QA1405500), the Natural Science Foundation of Shanghai Municipality (09ZR1426200), and the National Science Foundation for Young Scientists of China (30900808).

\section{References}

[1] W. Niu, S. Wu, Y. Zhang et al., "Validation of genetic association in apelin-AGTRL1 system with hypertension in a larger Han Chinese population," Journal of Hypertension, vol. 28, no. 9, pp. 1854-1861, 2010.

[2] D. J. Li, R. G. Evans, Z. W. Yang et al., "Dysfunction of the cholinergic anti-inflammatory pathway mediates organ damage in hypertension," Hypertension, vol. 57, pp. 298-307, 2011.

[3] G. Engström, "Inflammation, obesity and risk of hypertension: shared pathways or independent risk factors?" Journal of Human Hypertension, vol. 25, pp. 71-72, 2010.

[4] A. Agrotis, J. Saltis, and A. Bobik, "Transforming growth factor- $\beta 1$ gene activation and growth of smooth muscle from hypertensive rats," Hypertension, vol. 23, no. 5, pp. 593-599, 1994.

[5] P. Y. Mantel and C. B. Schmidt-Weber, "Transforming growth factor-beta: recent advances on its role in immune tolerance," Methods in Molecular Biology, vol. 677, pp. 303-338, 2011.

[6] C. Laviades, N. Varo, and J. Díez, "Transforming growth factor $\beta$ in hypertensives with cardiorenal damage," Hypertension, vol. 36, no. 4, pp. 517-522, 2000.

[7] A. Bobik, "Hypertension, transforming growth factor- $\beta$, angiotensin II and kidney disease," Journal of Hypertension, vol. 22, no. 7, pp. 1265-1267, 2004.

[8] R. Scaglione, C. Argano, G. Parrinello et al., "Relationship between transforming growth factor $\beta 1$ and progression of hypertensive renal disease," Journal of Human Hypertension, vol. 16, no. 9, pp. 641-645, 2002.

[9] C. Argano, G. Duro, S. Corrao et al., "Transforming growth factor $\beta 1$ T29C gene polymorphism and hypertension: relationship with cardiovascular and renal damage," Blood Pressure, vol. 17, no. 4, pp. 220-226, 2008.

[10] F. He, D. Zhao, F. Deng et al., "Association of TGF- $\beta 1$ gene polymorphisms in exon1 and blood levels with essential hypertension," Blood Pressure, vol. 19, no. 4, pp. 225-233, 2010.

[11] Y. Yamada, M. Fujisawa, F. Ando, N. Niino, M. Tanaka, and H. Shimokata, "Association of a polymorphism of the transforming growth factor- $\beta 1$ gene with blood pressure in Japanese individuals," Journal of Human Genetics, vol. 47, no. 5, pp. 243-248, 2002.

[12] V. F. Panoulas, K. M. J. Douglas, J. P. Smith et al., "Transforming growth factor- $\beta 1869 \mathrm{~T} / \mathrm{C}$, but not interleukin-6 -174G/ $\mathrm{C}$, polymorphism associates with hypertension in rheumatoid arthritis," Rheumatology, vol. 48, no. 2, pp. 113-118, 2009.

[13] W. Q. Niu, Y. Zhang, K. D. Ji, P. J. Gao, and D. L. Zhu, "Lack of association between $\alpha$-adducin G460W polymorphism and hypertension: evidence from a case-control study and a metaanalysis," Journal of Human Hypertension, vol. 24, no. 7, pp. 467-474, 2010.

[14] L. D. Cohn and B. J. Decker, "How meta-analysis increases statistical power," Psychological Methods, vol. 8, no. 3, pp. 243253, 2003.

[15] J. P. T. Higgins and S. G. Thompson, "Quantifying heterogeneity in a meta-analysis," Statistics in Medicine, vol. 21, no. 11, pp. 1539-1558, 2002.

[16] J. P. T. Higgins, S. G. Thompson, J. J. Deeks, and D. G. Altman, "Measuring inconsistency in meta-analyses," British Medical Journal, vol. 327, no. 7414, pp. 557-560, 2003.

[17] J. P. A. Ioannidis and T. A. Trikalinos, "Early extreme contradictory estimates may appear in published research: the Proteus phenomenon in molecular genetics research and randomized trials," Journal of Clinical Epidemiology, vol. 58, no. 6, pp. 543-549, 2005.

[18] M. Egger, G. D. Smith, M. Schneider, and C. Minder, "Bias in meta-analysis detected by a simple, graphical test," British Medical Journal, vol. 315, no. 7109, pp. 629-634, 1997.

[19] Y. S. Wei, Z. Li, and J. S. Lan, "Association of polymorphisms of transforming growth factor-beta 1 gene with susceptibility to essential hyperetnsion in Zhuang nationality of Guangxi province," Journal of the Fourth Military Medical University, vol. 26, pp. 341-344, 2005 (Chinese).

[20] B. C. Hu, S. L. Chu, J. G. Wang, G. L. Wang, P. J. Gao, and D. L. Zhu, "Single nucleotide polymorphisms of three candidate genes in essential hypertension," Chinese Journal of Internal Medicine, vol. 45, no. 4, pp. 281-284, 2006 (Chinese).

[21] Q. Y. Zhang, H. Dong, Y. Y. Wang et al., "The relationship of transforming growth factor-beta 1 gene polymorphisms and its plasma levels with hypertension and left ventricular hypertrophy," Chinese Journal of Geriatric, vol. 25, pp. 358360, 2006 (Chinese).

[22] C. L. Wei, Y. J. Wu, Q. Qiu, and H. Yang, "Study on the relation of transforming growth factor-beta 1 gene polymorphisms with essential hypertension," Chinese Journal of Geriatric, vol. 5, pp. 962-964, 2007 (Chinese).

[23] Z. D. Zhang, L. C. Chen, G. L. Zeng, Z. N. Tan, W. Deng, and J. Peng, "Study on the relationship between gene polymorphism of transforming growth factor- $\beta 1$ and atrial fibrillation in patients with essential hypertension," Journal of Clinical Research, vol. 26, pp. 211-213, 2009 (Chinese).

[24] G. Salanti, S. Sanderson, and J. P. T. Higgins, "Obstacles and opportunities in meta-analysis of genetic association studies," Genetics in Medicine, vol. 7, no. 1, pp. 13-20, 2005.

[25] A. Ruano-Ravina, M. Pérez-Ríos, and J. Miguel Barros-Dios, "Population-based versus hospital-based controls: are they comparable?” Gaceta Sanitaria, vol. 22, no. 6, pp. 609-613, 2008 . 
[26] W. Niu, Y. Qi, S. Guo, P. Gao, and D. Zhu, "Association of renin BglI polymphism with essential hypertension: a metaanalysis involving 1811 cases and 1626 controls," Clinical and Experimental Hypertension, vol. 32, no. 7, pp. 431-438, 2010.

[27] M. R. Munafò and J. Flint, "Meta-analysis of genetic association studies," Trends in Genetics, vol. 20, no. 9, pp. 439-444, 2004.

[28] T. V. Pereira, A. C. F. Nunes, M. Rudnicki, Y. Yamada, A. C. Pereira, and J. E. Krieger, "Meta-analysis of the association of 4 angiotensinogen polymorphisms with essential hypertension: a role beyond M235T?" Hypertension, vol. 51, no. 3, pp. 778783, 2008. 


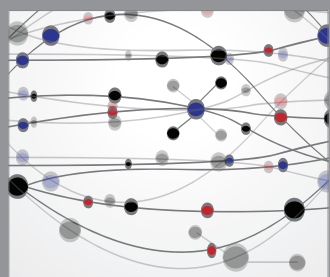

The Scientific World Journal
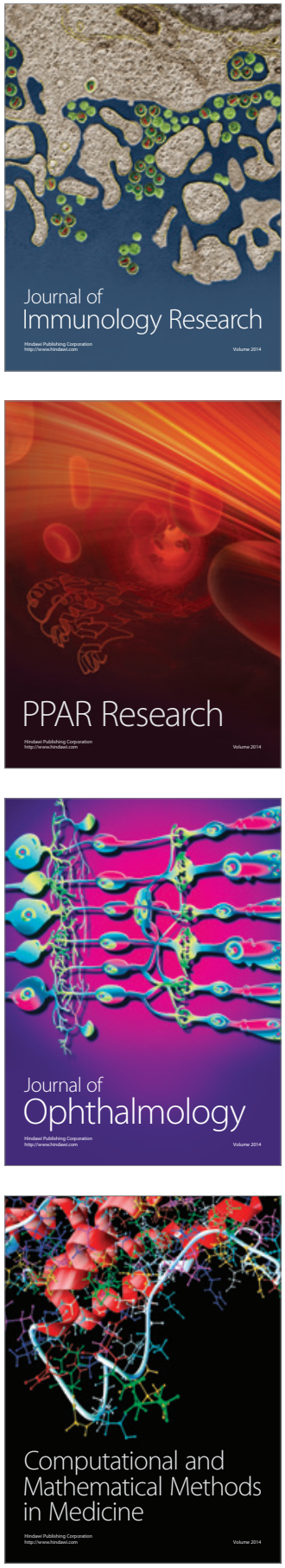

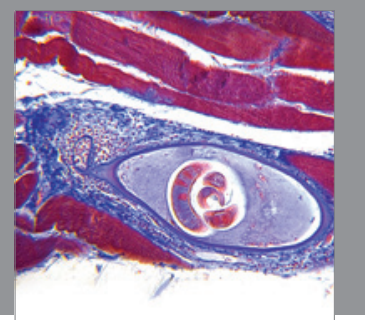

Gastroenterology

Research and Practice
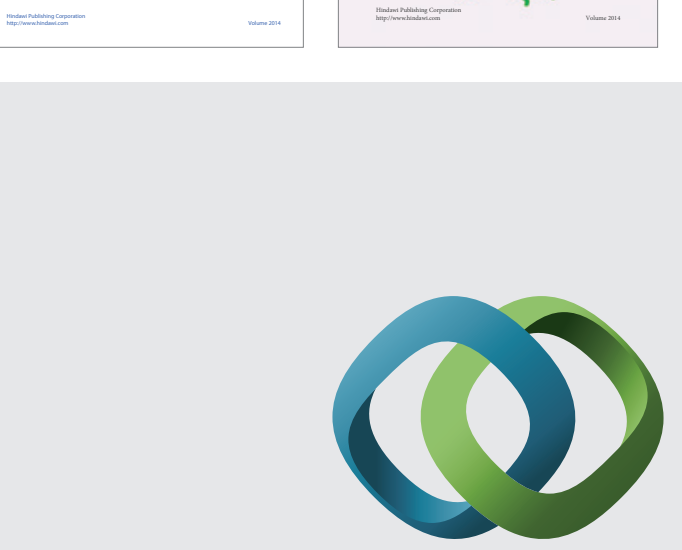

\section{Hindawi}

Submit your manuscripts at

http://www.hindawi.com
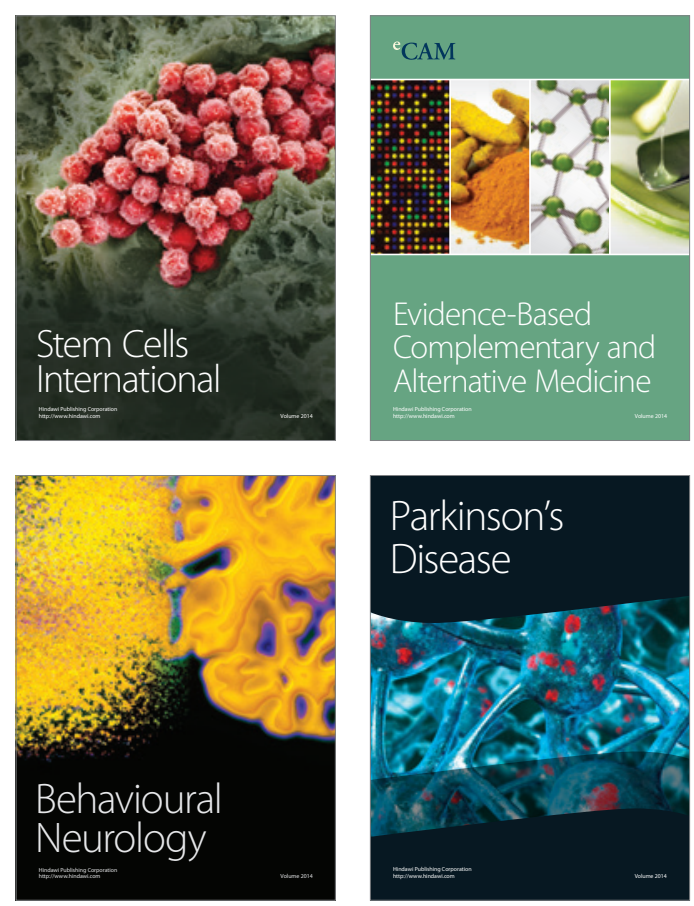

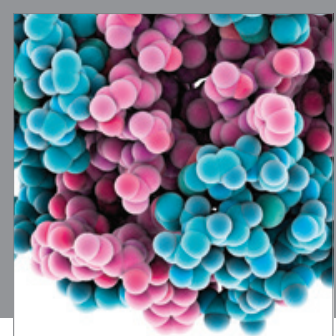

Journal of
Diabetes Research

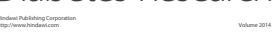

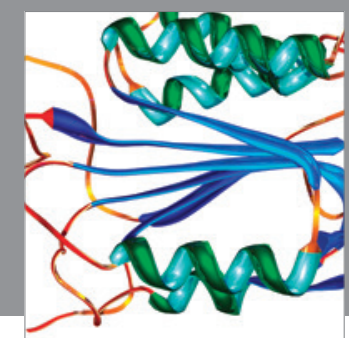

Disease Markers
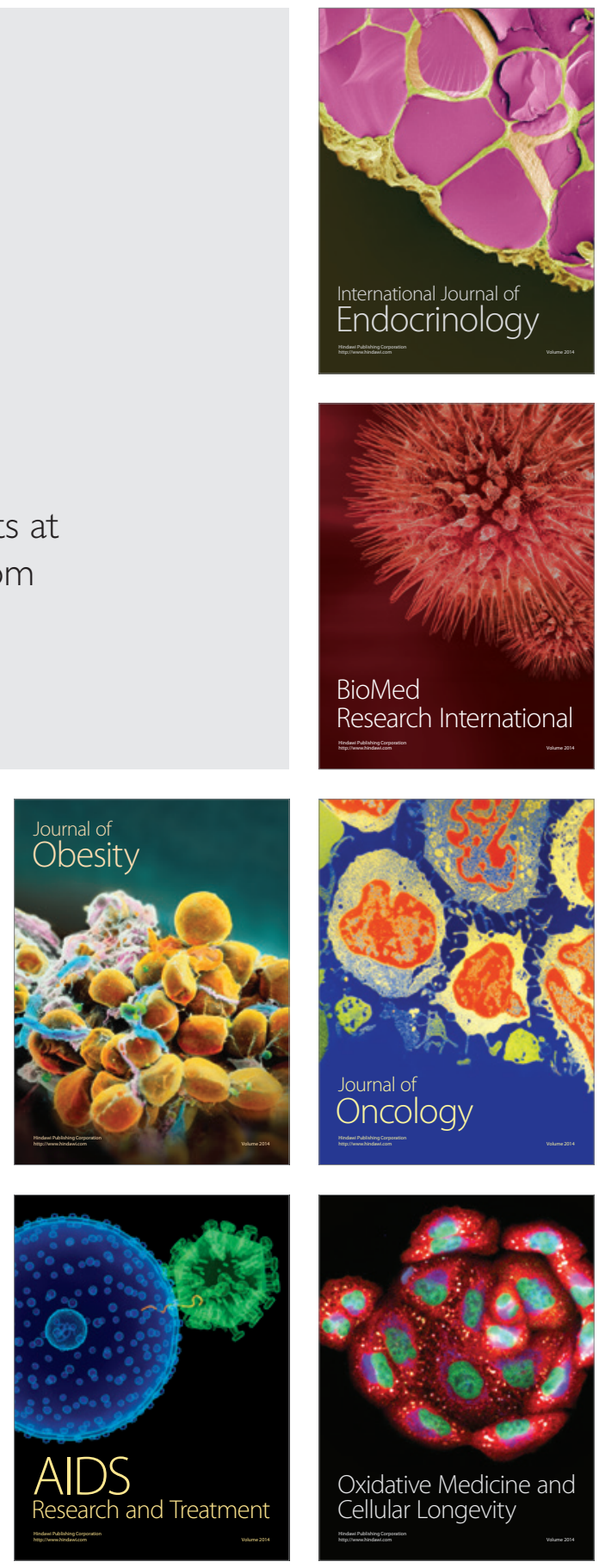